\title{
Three New Triterpenoids from European Mushroom Tricholoma terreum
}

\author{
Tao Feng $\cdot$ Juan He $\cdot$ Hong-Lian Ai $\cdot$ \\ Rong Huang $\cdot$ Zheng-Hui Li $\cdot$ Ji-Kai Liu
}

Received: 3 August 2015/Accepted: 1 September 2015/Published online: 9 September 2015

(C) The Author(s) 2015. This article is published with open access at Springerlink.com

\begin{abstract}
Three rare triterpenoids, saponaceolides Q-S (1-3), have been isolated from fruiting bodies of the mushroom Tricholoma terreum. Their structures were characterized based on extensive spectroscopic data. Compound 1 showed certain cytotoxicities against four human tumor cell lines.

Keywords Tricholoma terreum · Triterpenoids · Cytotoxicity
\end{abstract}

\section{Introduction}

Our previous work has identified Tricholoma terreum as a hitherto unknown poisonous European mushroom [1]. From which fifteen new triterpenoids terreolides A-F and saponaceolides H-P have been isolated. Terreolides A-F possessed novel frameworks, while saponaceolides B and $\mathrm{M}$ were the main toxins in the mushroom. The structural diversity, as well as important bioactivity discovery, prompted us to make a further study on this mushroom. According to an investigation on chloroform extract of $T$. Terreum collected in Arcachon in southwestern France,

Electronic supplementary material The online version of this article (doi:10.1007/s13659-015-0071-5) contains supplementary material, which is available to authorized users.

T. Feng $(\bowtie) \cdot$ J. He $\cdot$ R. Huang $\cdot$ Z.-H. Li $\cdot$ J.-K. Liu College of Pharmacy, South-Central University for Nationalities, Wuhan 430074, China

e-mail: fengtao@mail.kib.ac.cn

Z.-H. Li

e-mail: lizhenghui@mail.kib.ac.cn

J.-K. Liu

e-mail: jkliu@mail.kib.ac.cn

H.-L. Ai

College of Life Sciences, South-Central University for

Nationalities, Wuhan 430074, China three new triterpenoids, saponaceolides Q-S (1-3), have been obtained (Fig. 1). Their structures were established by extensive spectroscopic methods. Compounds 1-3 were evaluated for their cytotoxicities against five human cancer cell lines.

\section{Results and Discussion}

Compound 1 was isolated as a colorless oil. The HRESIMS data $\left(\mathrm{m} / z, 525.3193[\mathrm{M}+\mathrm{Na}]^{+}\right)$indicated the molecular formula $\mathrm{C}_{30} \mathrm{H}_{46} \mathrm{O}_{6}$, requiring eight degrees of unsaturation. The IR absorption bands at 3436 and $1723 \mathrm{~cm}^{-1}$ suggested the presence of hydroxy and carbonyl groups, respectively. In the ${ }^{1} \mathrm{H}$ NMR spectrum (Table 1), five singlets from $\delta_{\mathrm{H}}$ 0.54 to 1.29 were readily identified signals for five methyls, while a singlet at $\delta_{\mathrm{H}} 7.11(1 \mathrm{H}$, br $\mathrm{s}, \mathrm{H}-11)$ revealed the existence of a double bond that might have an $\alpha, \beta$-unsaturated keto moiety. The ${ }^{13} \mathrm{C}$ NMR and DEPT spectra displayed thirty carbon resonances that was classified into five $\mathrm{CH}_{3}$, thirteen $\mathrm{CH}_{2}$, four $\mathrm{CH}$, and eight $\mathrm{C}$, as shown in Table 2. These data displayed high similarities to those of saponaceolide $\mathrm{O}$, a triterpenoid isolated from the same mushroom by Yin et al. [1]. The key difference in $\mathbf{1}$ was that $\mathrm{C}-7$ was identified as a methylene $\left(\delta_{\mathrm{H}} 1.82\right.$ and 1.67 , $\left.\mathrm{m} ; \delta_{\mathrm{C}} 23.1\right)$ rather than a carbonyl carbon in saponaceolide $\mathrm{O}$, which was supported by ${ }^{1} \mathrm{H}-{ }^{1} \mathrm{H}$ COSY fragments of $\mathrm{H}-7$ 


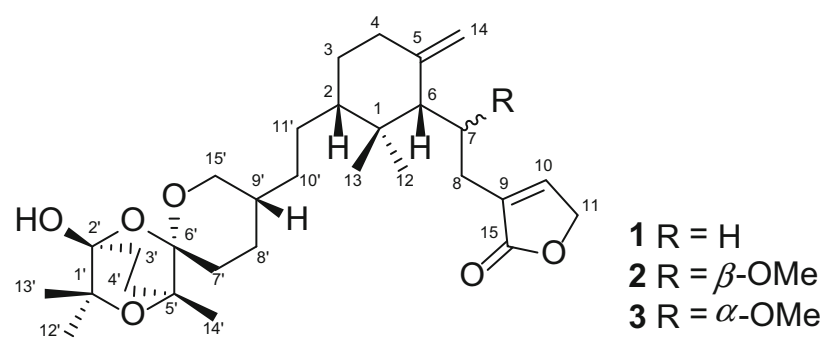

Fig. 1 Structures of compounds 1-3

with $\delta_{\mathrm{H}} 1.68(1 \mathrm{H}, \mathrm{m}, \mathrm{H}-6)$ and $2.48(1 \mathrm{H}, \mathrm{m}, \mathrm{H}-8 \mathrm{a})$, as well as $\mathrm{HMBC}$ correlations from $\mathrm{H}-7$ to $\delta_{\mathrm{C}} 53.5(\mathrm{~d}, \mathrm{C}-6)$ and 24.7 (t, C-8) (Fig. 2). Analyses of 2D NMR data suggested that the other parts of $\mathbf{1}$ were the same to those of saponaceolide $\mathrm{O}$ (Fig. 2) [1]. Compound 1 was, therefore, elucidated as shown in Fig. 1 and named saponaceolide Q.

Compound $\mathbf{2}$ was isolated as a colorless oil. The HRESIMS ion peak at $m / z 555.3290\left([\mathrm{M}+\mathrm{Na}]^{+}\right)$(calcd. 555.3292) indicated the molecular formula $\mathrm{C}_{31} \mathrm{H}_{48} \mathrm{O}_{7}$, requiring eight degrees of unsaturation. The ${ }^{1} \mathrm{H}$ and ${ }^{13} \mathrm{C}$ NMR spectra displayed similar patterns to those of $\mathbf{1}$ except for the signals at $\delta_{\mathrm{H}} 4.13(1 \mathrm{H}, \mathrm{dd}, J=7.6,5.2 \mathrm{~Hz}$,
H-7) and $3.30(3 \mathrm{H}, \mathrm{s}, \mathrm{MeO}-)$ and $\delta_{\mathrm{C}} 75.2(\mathrm{~d}, \mathrm{C}-7)$ and 57.2 (q, MeO-), suggesting the existence of a methoxy substituent. The HMBC correlation from $\delta_{\mathrm{H}} 3.30(3 \mathrm{H}, \mathrm{s}$, $\mathrm{MeO}-)$ to $\mathrm{C}-7$ indicated that a methoxy group placed at C-7, which was also supported by ${ }^{1} \mathrm{H}-{ }^{1} \mathrm{H}$ COSY correlations of $\mathrm{H}-7$ with $\delta_{\mathrm{H}} 1.60(1 \mathrm{H}, \mathrm{m}, \mathrm{H}-6)$ and $2.01(2 \mathrm{H}$, $\mathrm{m}, \mathrm{H}-8)$. Detailed analyses of $2 \mathrm{D}$ NMR data suggested that other parts of $\mathbf{2}$ were the same to those of $\mathbf{1}$. The coupling constant of $\mathrm{H}-7(J=7.6,5.2 \mathrm{~Hz})$ suggested an $S$ configuration of C-7 referring to the data of the literature [1-4]. Therefore, compound 2 was determined to be saponaceolide R.

The 1D NMR (Tables 1 and 2) and HRESIMS data of $\mathbf{3}$ were almost the same to those of $\mathbf{2}$, which informed that $\mathbf{3}$ might have the same framework to that of 2 . Detailed analyses of 2D NMR data suggested that $\mathbf{3}$ did possess the same planar structure to that of $\mathbf{2}$. However, tiny changes of NMR data of $\mathrm{CH}-6, \mathrm{CH}-7$, and $\mathrm{CH}_{2}-8$ suggested that the stereoconfiguration of $\mathrm{C}-7$ was different from that of 2 . The coupling constant of $\mathrm{H}-7(\mathrm{~d}, J=10.4 \mathrm{~Hz})$ was also significantly different to those reported previously (less than $8 \mathrm{~Hz}$ ) [1-4], which allowed an R configuration of C-7 in $\mathbf{3}$,

Table $1{ }^{1} \mathrm{H}$ NMR data for compounds $\mathbf{1}-\mathbf{3}(\delta$ in ppm, $J$ in $\mathrm{Hz})$

\begin{tabular}{|c|c|c|c|}
\hline Entry & 1 & 2 & 3 \\
\hline 2 & 1.09 overlapped & 1.06, overlapped & 1.15 overlapped \\
\hline 3 & $1.78 \mathrm{~m} ; 1.09 \mathrm{~m}$ & $1.76 \mathrm{~m} ; 1.07 \mathrm{~m}$ & $1.79 \mathrm{~m} ; 1.13 \mathrm{~m}$ \\
\hline 4 & $2.32 \mathrm{~m} ; 1.92 \mathrm{~m}$ & $2.29 \mathrm{~m} ; 1.88 \mathrm{~m}$ & $2.34 \mathrm{~m} ; 1.98 \mathrm{~m}$ \\
\hline 6 & $1.68 \mathrm{~m}$ & $1.60 \mathrm{~m}$ & $2.13 \mathrm{~m}$ \\
\hline 7 & $1.82 \mathrm{~m} ; 1.67 \mathrm{~m}$ & $4.13 \mathrm{dd}(7.6,5.2)$ & $4.10 \mathrm{~d}(10.4)$ \\
\hline 8 & $2.48 \mathrm{~m} ; 2.14 \mathrm{~m}$ & $2.01 \mathrm{~m}$ & $1.86 \mathrm{~m} ; 1.63 \mathrm{~m}$ \\
\hline 10 & 7.11 br s & 7.35 br s & 7.33 br s \\
\hline 11 & 4.78 br s & $4.84 \mathrm{~d}(6.0)$ & $4.84 \mathrm{~d}(17.0)$ \\
\hline 12 & $0.99 \mathrm{~s}$ & $0.94, \mathrm{~s}$ & $0.99, \mathrm{~s}$ \\
\hline 13 & $0.54 \mathrm{~s}$ & $0.53, \mathrm{~s}$ & $0.52, \mathrm{~s}$ \\
\hline 14 & 4.88 br s; 4.59 br s & 4.87, br s; 4.64, br s & 4.94, br s; 4.88, br s \\
\hline $3^{\prime}$ & $2.00 \mathrm{~m} ; 1.87 \mathrm{~m}$ & $2.00 \mathrm{~m} ; 1.87 \mathrm{~m}$ & $2.00 \mathrm{~m} ; 1.88 \mathrm{~m}$ \\
\hline $4^{\prime}$ & $2.16 \mathrm{~m} ; 1.68 \mathrm{~m}$ & $2.16 \mathrm{~m} ; 1.67$ & $2.17 \mathrm{~m} ; 1.67$ \\
\hline $7^{\prime}$ & $1.97 \mathrm{~m} ; 1.51 \mathrm{~m}$ & $1.96 \mathrm{~m} ; 1.50 \mathrm{~m}$ & $1.97 \mathrm{~m} ; 1.51 \mathrm{~m}$ \\
\hline $8^{\prime}$ & $1.67 \mathrm{~m}$ & $1.65 \mathrm{~m}$ & $1.67 \mathrm{~m}$ \\
\hline $9^{\prime}$ & $1.48 \mathrm{~m}$ & $1.47 \mathrm{~m}$ & $1.49 \mathrm{~m}$ \\
\hline $10^{\prime}$ & $1.26 \mathrm{~m} ; 1.03 \mathrm{~m}$ & $1.23 \mathrm{~m} ; 1.01 \mathrm{~m}$ & $1.26 \mathrm{~m} ; 1.04 \mathrm{~m}$ \\
\hline $11^{\prime}$ & $1.55 \mathrm{~m} ; 0.81 \mathrm{~m}$ & $1.53 \mathrm{~m} ; 0.79 \mathrm{~m}$ & $1.56 \mathrm{~m} ; 0.82 \mathrm{~m}$ \\
\hline $12^{\prime}$ & $1.29 \mathrm{~s}$ & $1.29 \mathrm{~s}$ & $1.29 \mathrm{~s}$ \\
\hline $13^{\prime}$ & $1.21 \mathrm{~s}$ & $1.21 \mathrm{~s}$ & $1.22 \mathrm{~s}$ \\
\hline $14^{\prime}$ & $1.09 \mathrm{~s}$ & $1.09 \mathrm{~s}$ & $1.10 \mathrm{~s}$ \\
\hline \multirow[t]{2}{*}{$15^{\prime}$} & $3.69 \mathrm{dd}(11.0,10.9)$ & $3.68 \mathrm{dd}(11.0,10.8)$ & $3.69 \mathrm{dd}(11.3,10.8)$ \\
\hline & $3.60 \mathrm{dd}(11.0,4.3)$ & $3.58 \mathrm{dd}(11.0,4.2)$ & $3.60 \mathrm{dd}(11.3,4.0)$ \\
\hline $\mathrm{MeO}-$ & & $3.30 \mathrm{~s}$ & $3.30 \mathrm{~s}$ \\
\hline
\end{tabular}

Data $(\delta)$ were measured in $\mathrm{CDCl}_{3}$. The assignments were based on DEPT, ${ }^{1} \mathrm{H}-{ }^{1} \mathrm{H}$ COSY, HSQC, and HMBC experiments 
Table $2{ }^{13} \mathrm{C}$ NMR data for compounds $\mathbf{1}-\mathbf{3}$ ( $\delta$ in ppm)

\begin{tabular}{|c|c|c|c|}
\hline No. & 1 & 2 & 3 \\
\hline 1 & $39.8 \mathrm{C}$ & $39.7 \mathrm{C}$ & $39.5 \mathrm{C}$ \\
\hline 2 & $48.0 \mathrm{CH}$ & $47.9 \mathrm{CH}$ & $48.1 \mathrm{CH}$ \\
\hline 3 & $30.3 \mathrm{CH}_{2}$ & $29.1 \mathrm{CH}_{2}$ & $30.5 \mathrm{CH}_{2}$ \\
\hline 4 & $37.3 \mathrm{CH}_{2}$ & $37.2 \mathrm{CH}_{2}$ & $37.5 \mathrm{CH}_{2}$ \\
\hline 5 & $148.0 \mathrm{C}$ & $148.8 \mathrm{C}$ & $148.1 \mathrm{C}$ \\
\hline 6 & $53.5 \mathrm{CH}$ & $49.2 \mathrm{CH}$ & $49.3 \mathrm{CH}$ \\
\hline 7 & $23.1 \mathrm{CH}_{2}$ & $75.2 \mathrm{CH}$ & $75.4 \mathrm{CH}$ \\
\hline 8 & $24.7 \mathrm{CH}_{2}$ & $30.5 \mathrm{CH}_{2}$ & $32.0 \mathrm{CH}_{2}$ \\
\hline 9 & $134.8 \mathrm{C}$ & $135.0 \mathrm{C}$ & $136.5 \mathrm{C}$ \\
\hline 10 & $143.8 \mathrm{CH}$ & $146.8 \mathrm{CH}$ & $144.9 \mathrm{CH}$ \\
\hline 11 & $70.1 \mathrm{CH}_{2}$ & $70.2 \mathrm{CH}_{2}$ & $70.6 \mathrm{CH}_{2}$ \\
\hline 12 & $26.5 \mathrm{CH}_{3}$ & $26.4 \mathrm{CH}_{3}$ & $26.6 \mathrm{CH}_{3}$ \\
\hline 13 & $14.9 \mathrm{CH}_{3}$ & $14.9 \mathrm{CH}_{3}$ & $15.3 \mathrm{CH}_{3}$ \\
\hline 14 & $106.7 \mathrm{CH}_{2}$ & $106.5 \mathrm{CH}_{2}$ & $107.9 \mathrm{CH}_{2}$ \\
\hline 15 & $174.4 \mathrm{C}$ & $172.8 \mathrm{C}$ & $173.2 \mathrm{C}$ \\
\hline $1^{\prime}$ & $77.5 \mathrm{C}$ & $77.5 \mathrm{C}$ & $77.7 \mathrm{C}$ \\
\hline $2^{\prime}$ & $96.6 \mathrm{C}$ & $96.6 \mathrm{C}$ & $96.8 \mathrm{C}$ \\
\hline $3^{\prime}$ & $27.9 \mathrm{CH}_{2}$ & $27.9 \mathrm{CH}_{2}$ & $28.1 \mathrm{CH}_{2}$ \\
\hline $4^{\prime}$ & $28.5 \mathrm{CH}_{2}$ & $28.5 \mathrm{CH}_{2}$ & $28.4 \mathrm{CH}_{2}$ \\
\hline $5^{\prime}$ & $72.8 \mathrm{C}$ & $72.8 \mathrm{C}$ & $73.0 \mathrm{C}$ \\
\hline $6^{\prime}$ & $101.4 \mathrm{C}$ & $101.2 \mathrm{C}$ & $101.5 \mathrm{C}$ \\
\hline $7^{\prime}$ & $29.2 \mathrm{CH}_{2}$ & $29.2 \mathrm{CH}_{2}$ & $29.5 \mathrm{CH}_{2}$ \\
\hline $8^{\prime}$ & $24.8 \mathrm{CH}_{2}$ & $24.8 \mathrm{CH}_{2}$ & $25.1 \mathrm{CH}_{2}$ \\
\hline $9^{\prime}$ & $35.7 \mathrm{CH}$ & $35.6 \mathrm{CH}$ & $35.9 \mathrm{CH}$ \\
\hline $10^{\prime}$ & $31.7 \mathrm{CH}_{2}$ & $31.6 \mathrm{CH}_{2}$ & $31.9 \mathrm{CH}_{2}$ \\
\hline $11^{\prime}$ & $27.6 \mathrm{CH}_{2}$ & $27.6 \mathrm{CH}_{2}$ & $28.1 \mathrm{CH}_{2}$ \\
\hline $12^{\prime}$ & $25.9 \mathrm{CH}_{3}$ & $25.9 \mathrm{CH}_{3}$ & $26.1 \mathrm{CH}_{3}$ \\
\hline $13^{\prime}$ & $22.4 \mathrm{CH}_{3}$ & $22.4 \mathrm{CH}_{3}$ & $22.6 \mathrm{CH}_{3}$ \\
\hline $14^{\prime}$ & $20.9 \mathrm{CH}_{3}$ & $20.9 \mathrm{CH}_{3}$ & $21.1 \mathrm{CH}_{3}$ \\
\hline $15^{\prime}$ & $65.8 \mathrm{CH}_{2}$ & $65.9 \mathrm{CH}_{2}$ & $66.2 \mathrm{CH}_{2}$ \\
\hline $\mathrm{MeO}-$ & & $57.2 \mathrm{CH}_{3}$ & $58.4 \mathrm{CH}_{3}$ \\
\hline
\end{tabular}

Data $(\delta)$ were measured in $\mathrm{CDCl}_{3}$. The assignments were based on DEPT, ${ }^{1} \mathrm{H}^{-1} \mathrm{H}$ COSY, HSQC, and HMBC experiments

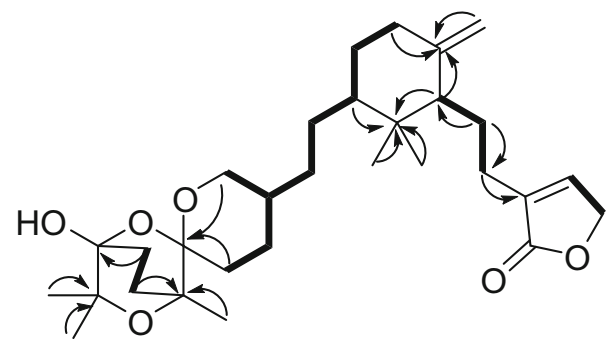

Fig. 2 Key 2D NMR correlations of saponaceolide R (2)

that was also in agreement with those described in the literature [3]. Therefore, compound $\mathbf{3}$ was established and named saponaceolide $\mathrm{S}$.
Table 3 Cytotoxicities of compounds $\mathbf{1 - 3}\left(\mathrm{IC}_{50}, \mu \mathrm{M}\right)$

\begin{tabular}{llllll}
\hline Entry & HL-60 & SMMC-7721 & A-549 & MCF-7 & SW480 \\
\hline $\mathbf{1}$ & 12.2 & 19.3 & $>40$ & 12.2 & 1.4 \\
$\mathbf{2}$ & $>40$ & $>40$ & $>40$ & $>40$ & $>40$ \\
$\mathbf{3}$ & $>40$ & $>40$ & $>40$ & $>40$ & $>40$ \\
Cisplatin & 2.4 & 11.2 & 17.6 & 18.7 & 14.9 \\
\hline
\end{tabular}

Many triterpenoids in this type, such as saponaceolides $\mathrm{B}, \mathrm{E}$, and $\mathrm{F}$, have been reported to possess cytotoxicities to several cancer cell lines [2-4]. Compounds 1-3 were, therefore, evaluated for their cytotoxicities to five human cancer cell lines. As a result, compound 1 showed moderate activities as shown in Table 3.

\section{Experimental}

\subsection{General Experimental Procedures}

Optical rotations were measured on a Jasco-P-1020 polarimeter. IR spectra were obtained using a Bruker Tensor 27 FT-IR spectrometer with $\mathrm{KBr}$ pellets. NMR spectra were acquired with instrument of a Bruker DRX600 with tetramethylsilane (TMS) used as an internal standard at room temperature. HRESIMS were recorded on an API QSTAR pulsar spectrometer. Silica gel (200-300 mesh), Sephadex LH-20 and RP-18 gel $(20-45 \mu \mathrm{m})$ were used for column chromatography (CC). Fractions were monitored by thin layer chromatography and spots were visualized by heating silica gel plates immersed in $\mathrm{H}_{2} \mathrm{SO}_{4}$ in $\mathrm{EtOH}$, in combination with the Agilent 1200 series HPLC system (Eclipse XDB-C18 column, $5 \mu \mathrm{m}$, $4.6 \times 150 \mathrm{~mm})$.

\subsection{Mushroom Material}

Wild mushrooms, T. terreum, were collected from Arcachon in southwestern France in December 2012 and identified by Prof. Zhu-Liang Yang of Kunming Institute of Botany, Chinese Academy of Sciences. A specimen (No. KIB20121205.2) was deposited at the Kunming Institute of Botany, Chinese Academy of Sciences. For details of this mushroom please see that reported previously [1].

\subsection{Extraction and Isolation}

The fresh fruiting bodies of $T$. Terreum $(3 \mathrm{~kg})$ were extracted with chloroform $(24 \mathrm{~h} \times 3)$, and then partitioned with water (1:1). Finally, a chloroform extract (12 g) was 
obtained, which was submitted to silica gel CC using petroleum-acetone (from 1:0 to $0: 1$ ) to give six fractions (A-F). Fraction B (1.8 g) was separated by reverse-phased $\mathrm{CC}$ eluted with gradient mixture of $\mathrm{MeOH}$ and $\mathrm{H}_{2} \mathrm{O}$ (30:70-100:0, v/v) to afford five sub-fractions (B1-B5). Fraction B2 (32 mg) was purified by Sephadex LH-20 $(\mathrm{MeOH})$ to give compound $\mathbf{1}(2.2 \mathrm{mg})$, while fraction B4 (57 mg) was also purified by Sephadex LH-20 (MeOH) to give compounds $2(2.3 \mathrm{mg})$ and $3(1.3 \mathrm{mg})$.

\subsubsection{Saponaceolide $Q(\mathbf{1})$}

Colorless oil, $\alpha_{\mathrm{D}}^{23}+7.2(c 0.12 \mathrm{MeOH})$; IR $(\mathrm{KBr}) v_{\max }$ 3436, 2937, 1723, 1448, 1367, 1201, 1068, $991 \mathrm{~cm}^{-1}$; for ${ }^{1} \mathrm{H}(600 \mathrm{MHz})$ and ${ }^{13} \mathrm{C} \mathrm{NMR}(150 \mathrm{MHz})$ data $\left(\mathrm{CDCl}_{3}\right)$, see Tables 1 and 2, respectively; HRESIMS: $m / z \quad 525.3193$ (calcd for $\mathrm{C}_{30} \mathrm{H}_{46} \mathrm{O}_{6} \mathrm{Na},[\mathrm{M}+\mathrm{Na}]^{+}, 525.3187$ ).

\subsubsection{Saponaceolide $R(2)$}

Colorless oil, $\alpha_{\mathrm{D}}^{23}+19.7(c 0.10 \mathrm{MeOH})$; IR $(\mathrm{KBr}) v_{\max }$ 3443, 2926, 1726, 1457, 1381, 1065, $998 \mathrm{~cm}^{-1}$; for ${ }^{1} \mathrm{H}$ $(600 \mathrm{MHz})$ and ${ }^{13} \mathrm{C} \mathrm{NMR}(150 \mathrm{MHz})$ data $\left(\mathrm{CDCl}_{3}\right)$, see Tables 1 and 2, respectively; HRESIMS: $\mathrm{m} / \mathrm{z} 555.3290$ (calcd for $\mathrm{C}_{31} \mathrm{H}_{48} \mathrm{O}_{7} \mathrm{Na},[\mathrm{M}+\mathrm{Na}]^{+}$, 555.3292).

\subsubsection{Saponaceolide $S$ (3)}

Colorless oil, $\alpha_{\mathrm{D}}^{23}+13.7(c 0.11 \mathrm{MeOH})$; IR $(\mathrm{KBr}) v_{\max }$ 3441, 2926, 1724, 1452, 1382, 1120, $997 \mathrm{~cm}^{-1}$; for ${ }^{1} \mathrm{H}$ $(600 \mathrm{MHz})$ and ${ }^{13} \mathrm{C} \mathrm{NMR}(150 \mathrm{MHz})$ data $\left(\mathrm{CDCl}_{3}\right)$, see Tables 1 and 2, respectively; HRESIMS: $\mathrm{m} / \mathrm{z} 555.3290$ (calcd for $\mathrm{C}_{31} \mathrm{H}_{48} \mathrm{O}_{7} \mathrm{Na},[\mathrm{M}+\mathrm{Na}]^{+}$, 555.3292).

\subsection{Cytotoxicity Assay}

Human myeloid leukemia HL-60, hepatocellular carcinoma SMMC-7721, lung cancer A-549 cells, breast cancer
MCF-7 and colon cancer SW480 cell lines were used in the cytoxic assay. All cell lines were cultured in RPMI-1640 or DMEM medium (Hyclone, USA), supplemented with $10 \%$ fetal bovine serum (Hyclone, USA) in $5 \% \mathrm{CO}_{2}$ at $37{ }^{\circ} \mathrm{C}$. The cytotoxicity assay was performed according to the MTT (3-(4,5-dimethylthiazol-2-yl)-2,5-diphenyl tetrazolium bromide) method in 96-well microplates [5]. Cisplatin was used as a positive control.

Acknowledgments This project was supported by the National Natural Sciences Foundation of China (U1132607, 81102346).

\section{Compliance with ethical standards}

Conflict of interest The authors declare no conflict of interest.

Open Access This article is distributed under the terms of the Creative Commons Attribution 4.0 International License (http:// creativecommons.org/licenses/by/4.0/), which permits unrestricted use, distribution, and reproduction in any medium, provided you give appropriate credit to the original author(s) and the source, provide a link to the Creative Commons license, and indicate if changes were made.

\section{References}

1. X. Yin, T. Feng, J.H. Shang, Y.L. Zhao, F. Wang, Z.H. Li, Z.J. Dong, X.D. Luo, J.K. Liu, Chem. Eur. J. 20, 7001-7009 (2014)

2. M. De Bernardi, L. Garlaschelli, G. Gatti, G. Vidari, P. Vita-Finzi, Tetrahedron 44, 235-240 (1988)

3. M. De Bernardi, L. Garlaschelli, L. Toma, G. Vidari, P. VitaFinza, Tetrahedron 47, 7109-7116 (1991)

4. K. Yoshikawa, M. Kuroboshi, S. Arihara, N. Miura, N. Tujimura, K. Sakamoto, Chem. Pharm. Bull. 50, 1603-1606 (2002)

5. T.J. Mosmann, J. Immunol. Methods 65, 55-63 (1983) 